\title{
Interference of E2-2-mediated effect in endothelial cells by FAM96B through its limited expression of E2-2
}

\author{
Weiwen Yang ${ }^{1,5}$, Fumiko Itoh ${ }^{1,2,5}$, Hirotoshi Ohya ${ }^{1}$, Fukiko Kishimoto ${ }^{1}$, Aya \\ Tanaka $^{1}$, Naoko Nakano ${ }^{1,3,4}$, Susumu Itoh ${ }^{1,3,4,6}$, and Mitsuyasu Kato ${ }^{1}$ \\ ${ }^{1}$ Department of Experimental Pathology, Graduate School of Comprehensive Human \\ Sciences, University of Tsukuba, 1-1-1 Tennodai, Tsukuba, Ibaraki 305-8575, Japan, \\ ${ }^{2}$ Laboratory of Cardiovascular Medicine, Tokyo University of Pharmacy and Life \\ Sciences, 1432-1, Horinouchi, Hachioji, Tokyo 192-0392, Japan, ${ }^{3}$ Laboratory of \\ Biochemistry, and ${ }^{4}$ High Technology Research Center, Showa Pharmaceutical University, \\ 3-3165 Higashi-Tamagawagakuen, Machida, Tokyo 194-8543, Japan.
}

\section{Running title: Limited expression of E2-2 by FAM96B}

Keywords: E2-2; endothelial cell; FAB96B; migration; VEGFR2

Word count: 4256 words

Table: 0 table

Figure: 5 figures

Supporting information: 1 supplementary table, 1 supplementary figure legend and 5 supplementary figures

${ }^{5}$ Contributed equally.

${ }^{6}$ Corresponding author:

Susumu Itoh

Laboratory of Biochemistry,

Showa Pharmaceutical University,

3-3165 Higashi-Tamagawagakuen,

Machida, Tokyo 194-8543, Japan

Tel: +81-42-721-1558

Fax: +81-42-721-1588

Email: sitoh@ac.shoyaku.ac.jp 


\section{Summary}

The basic helix-loop-helix (bHLH) protein E2-2 is known to play a role in quiescence of endothelial cells (ECs). However, how the activity of E2-2 is controlled in the cells is still unclear. In this study, we identified FAM96B as an interaction partner of E2-2. FAM96B interfered with E2-2-mediated effects on luciferase reporter activities. Furthermore, the suppression of VEGFR2 promoter activity by E2-2 was rescued by the expression of FAM96B in a dose-dependent manner. Interestingly, FAM96B decreased the expression of ectopic and endogenous E2-2 proteins. Mutational analysis revealed that the middle region of FAM96B is required for the limited expression of E2-2 protein. When FAM96B was expressed in ECs, the EC migration, proliferation and tube formation were potentiated. Taken together, these findings suggest that FAM96B acts as a regulator of E2-2 via the control of its protein expression. 


\section{Introduction}

The basic-helix-loop-helix (bHLH) protein E2-2, alternatively termed ITF2, TCF4, SEF2, and SEF2-1B, was originally identified as a transcription factor that binds to the $\mu \mathrm{E} 5$ and $\kappa \mathrm{E} 2$ motifs in the immunoglobulin heavy and light chain enhancers, respectively (1). Together with E12/47 and HEB, E2-2 belongs to the E protein family. E proteins are widely expressed in tissues and bind to consensus DNA sequences containing (G/A)CAXXTG(G/A) as either homodimers or heterodimers with other HLH family molecules $(2,3)$. The E protein family is known to regulate lymphocyte development (4), neural differentiation (5), and myogenesis (6). Recently, we and another group demonstrated that E proteins also regulate blood vessel formation (7-9).

The activity of E proteins is known to be controlled via their interaction with other HLH proteins (10), coactivators $(11,12)$, or corepressors (13) as well as via their own phosphorylation (14). The Id family, which lacks the basic region important for DNA binding activity, associates with E proteins to inhibit their DNA binding activity (10). The coactivators CBP and p300 interact with E proteins via their AD1 domain to potentiate their transcriptional activity $(11,12)$, although the ETO family blocks the transcriptional activity of E proteins via its direct interaction (13).

Angiogenesis, the formation of new blood vessels, is crucial for vascular development and homeostasis. Aberrant vascularization leads us to a number of diseases including atherosclerosis, tumorigenicity, and retinopathy. Angiogenesis occurs by sprouting of new vessels from preexisting ones or by intussusceptive microvascular growth. Thus, angiogenesis is essential during embryonic development as well as in adulthood. In general, vascular formation is quiet in adulthood although angiogenesis 
involved in wound healing, inflammation, ischemia, and the female reproductive cycle has been observed (15). Angiogenesis is divided into two phases: the activation phase and the resolution phase. The angiogenic switch depends on a finely tuned balance between stimulators (VEGF, FGF-2, angiopoietins, hypoxia, and so on) and inhibitors (angiostatin, endostatin, interferon- $\alpha$, and so on). During the activation phase, proliferation of endothelial cells (ECs), increase of vascular permeability, and degradation of extracellular matrix components can be observed. Consequently, ECs make new capillary sprouts. During the resolution phase, the proliferation and migration of ECs stops, whereafter the basement membrane is reconstituted and the vessels mature (16). In our recent studies, E2-2 as well as E2A inhibited the activity of the VEGFR2 promoter to inhibit the activity of endothelium in vivo and in vitro. In contrast, Id1 and SCL/TAL1, both of which possess the HLH domains, relieved E2-2-mediated inhibition of VEGFR2 promoter activity because of heterodimer formation with E2-2 $(7,8)$. However, partner(s) other than HLH-containing proteins can still regulate the activity of E2-2.

To gain more insight into the molecular mechanisms by which E2-2 suppresses the activity of endothelium, we surveyed its interaction partner(s) and identified FAM96B, alternatively named CGI-128. However, its function has yet to be identified. In this study, we explored the regulatory role of FAM96B on the function of E2-2. In addition, we examined its function in EC activation. We found that FAM96B promoted decrease of E2-2 protein to rescue E2-2-mediated repression of VEGFR2 promoter activity. Consequently, FAM96B concomitantly enhanced EC migration, proliferation and tube formation. 


\section{Material and Methods}

\section{$\underline{\text { Plasmids and adenoviruses }}$}

The cDNA for human FAM96B was cloned by RT-PCR and sequenced to be verified prior to use. FAM96B(42-121) and FAM96B $\Delta$ (42-121) were generated by Pfx DNA polymerase (Invitrogen) using human FAM96B as the template. Subsequently, each DNA was ligated into either Flag-pDEF3 or Myc-pDEF3 (17). Myc-E2-2 and Flag-E2-2 have been previously described (8). MCKpfos-luc was generously provided by Dr. M. Sigvardsson (18), and pGL2b-VEGFR2-luc (-166bp/+267bp) by Dr. C. C. W. Hughes (19). Adenovirus expressing Myc-FAM96B was generated using the pAdTrack-CMV vector (20). After pAdTrack-CMV-Myc-FAM96B was recombined with pAdEasy-1 (20), the resulting plasmid was transfected into $293 \mathrm{~T}$ cells, and the adenoviruses were amplified. LacZ- and constitutively active activin receptor-like kinase 6 (ALK6ca)/HA-expressing adenoviruses are previously described (8).

\section{Cell culture}

COS7 cells, NIH3T3 cells, and mouse embryonic endothelial cells (MEECs) (21) were maintained in Dulbecco’s modified Eagle’s media (DMEM, Sigma) containing 10\% fetal calf serum (FCS, Gibco), 1X MEM nonessential amino acids (NEAA, Invitrogen), and $100 \mathrm{U} / \mathrm{mL}$ penicillin/streptomycin. Calf pulmonary artery epithelial cells (CPAEs) (22) were cultured in DMEM with 10\% FCS, 20 mM HEPES, and 100 U/mL penicillin/streptomycin. Primary human umbilical vein endothelial cells (HUVECs) were cultured in EBM (TAKARA) supplemented with 2\% FCS. MEECs and HUVECs were grown on $0.1 \%$ gelatin-coated dishes. 


\section{$\underline{\text { Adenoviral infections }}$}

Adenoviruses were incubated in DMEM containing polybrene $(80 \mu \mathrm{g} / \mathrm{mL})$ for $2 \mathrm{~h}$ and added to the dishes. Two hours after infection, the cells were washed and allowed to recover for $24 \mathrm{~h}$ prior to the experiments.

\section{Immunoprecipitation and Western blotting}

To detect interactions among proteins, plasmids were transfected into COS7 cells $\left(5 \times 10^{5}\right.$ cells/6-cm dish) using FuGENE6 (Roche). Forty hours after the transfection, the cells were lysed in $500 \mu \mathrm{L}$ of TNE buffer (10 mM Tris [pH 7.4], $150 \mathrm{mM} \mathrm{NaCl,} 1 \mathrm{mM}$ ethylenediamine- $N^{\prime}, N^{\prime}, N^{\prime}, N^{\prime}$-tetraaccetic acid [EDTA], 1\% NP-40, $1 \mathrm{mM}$ phenylmethylsulfonyl-l-fluoride [PMSF], $5 \mu \mathrm{g} / \mathrm{mL}$ leupeptin, $100 \mathrm{U} / \mathrm{mL}$ aprotinin, $2 \mathrm{mM}$ sodium vanadate, $40 \mathrm{mM} \mathrm{NaF}$, and $20 \mathrm{mM} \beta$-glycerophosphate). The cell lysates were precleared with protein $\mathrm{G}$ sepharose beads (GE Healthcare) for 30 min at $4^{\circ} \mathrm{C}$ and then incubated with anti-Flag M5 (Sigma), anti-Myc9E10 (SantaCruz), or anti-E2-2 (M03) (Abnova) antibody for $2 \mathrm{~h}$ at $4^{\circ} \mathrm{C}$. Protein complexes were immunoprecipitated by incubation with protein G Sepharose beads for 30 min at $4^{\circ} \mathrm{C}$ and then washed three times with TNE buffer. The immunoprecipitated proteins and aliquots of the total cell lysates were boiled for 5 min in sample buffer, separated by SDS-polyacrylamide gel electrophoresis (SDS-PAGE), and transferred to Hybond-C Extra membrane (GE Healthcare). The membranes were probed with anti-Myc9E10 or anti-Flag M5 antibody. Primary antibodies were detected with horseradish peroxidase-conjugated goat anti-mouse antibody (GE Healthcare) and chemiluminescent substrate (Pierce). The protein expression in the total cell lysates was evaluated by Western blotting using anti-Flag M5, anti-Myc9E10, or anti-E2-2 (M03) antibody. As internal controls, the 
expressions of $\beta$-actin and GFP were detected by using anti- $\beta$-actin antibody (Sigma) and anti-GFP (SantaCruz) antibody, respectively.

\section{Immunofluorescence}

MEECs were grown on glass coverslips coated with $0.1 \%$ gelatin one day prior to the transfection. Cotransfection of Flag-E2-2 with Myc-FAM96B was performed using Lipofectamine ${ }^{\mathrm{TM}}$ (Invitrogen) and Plus ${ }^{\mathrm{TM}}$ Reagent (Invitrogen). After 40 h, the slips were washed with PBS, fixed with 4\% paraformaldehyde (PFA) for 10 min, washed three times with PBS, permeabilized with 0.1\% Triton X-100 (Sigma) for 10 min, and blocked with 5\% normal swine serum in PBS for $1 \mathrm{~h}$ at $37^{\circ} \mathrm{C}$. Mouse anti-E2-2 (M03) (1:250) and rabbit anti-Myc (1:250) (Upstate Biotechnology) antibodies in 5\% normal swine serum in PBS were added and incubated for $1 \mathrm{~h}$ at $37^{\circ} \mathrm{C}$. The slips were washed three times with PBS and then incubated with Alexa488-conjugated goat anti-mouse IgG (Molecular Probes) or Alexa555-conjugated-goat anti-rabbit IgG (Molecular Probes) at 1:250 for $1 \mathrm{~h}$ at room temperature. After the nuclei were stained with $2 \mu \mathrm{g} / \mathrm{mL}$ DAPI for $10 \mathrm{~min}$, the slips were washed three times with distilled water, and the fluorescent signals visualized by microscopy (Zeiss).

\section{Transcriptional reporter assay}

CPAEs were seeded at $5 \times 10^{4}$ cells/well in 12-well plates one day prior to the transfection. The cells were transfected using Lipofectamine ${ }^{\mathrm{TM}}$ and Plus ${ }^{\mathrm{TM}}$ Reagent. After $40 \mathrm{~h}$ of transfection, the lysates were prepared and the luciferase activity was measured using a luciferase assay system (Promega). The results were corrected for $\beta$-galactosidase activity (pCH110; GE Healthcare). Each experment was carried out in triplicate and repeated at least twice. Values are presented as means $\pm S$. D. $(n=3)$ 


\section{Migration assay}

Cell migration assays were performed using a Boyden chamber. Costar nucleopore filters (8- $\mu \mathrm{m}$ pore) were coated with $10 \mu \mathrm{g} / \mathrm{mL}$ fibronectin (Sigma) overnight at $4^{\circ} \mathrm{C}$. The chambers were washed three times with PBS. Adenovirus-infected HUVECs starved for $12 \mathrm{~h}$ without FCS were added to the top of each migration chamber at a density of $2.5 \mathrm{x}$ $10^{5}$ cells/chamber in $150 \mu \mathrm{L}$ of EBM without FCS. The cells were allowed to migrate to the underside of the chamber. After $24 \mathrm{~h}$, the cells were fixed in 4\% PFA and stained with $0.5 \%$ crystal violet (dissolved in $25 \%$ methanol). The upper surface was wiped with a cotton swab to remove non-migrating cells. Cells present on the lower surface were counted. Each experiment was carried out in triplicate and repeated a few times. Values are presented as means $\pm S$. D. ( $n=3)$.

Network formation assay

HUVECs $\left(2 \times 10^{4}\right.$ cells/well in an 8-well Lab-Tek ${ }^{\circledR}$ chamber; ThermoFisher) infected with adenoviruses were seeded on growth factor-reduced Matrigel (BD Biosciences, San Jose, CA). Images were captured $6 \mathrm{~h}$ and $12 \mathrm{~h}$ after ECs were seeded (8). The total area of tube-like structures formed by HUVECs in each well was measured using Image J from NIH.

\section{Cell proliferation}

Proliferation was measured by the incorporation of $\left[{ }^{3} \mathrm{H}\right]$-thymidine to HUVECs. HUVECs were seeded in 24-well plates one day before adenoviral infection. Values are presented as means \pm S. D. $(\mathrm{n}=3)$.

$\underline{\text { RNA isolation and RT-PCR }}$ 
Total RNA was isolated using the RNeasy kit (QIAGEN, Valencia, CA). Reverse transcription was carried out by using a First-Strand cDNA Synthesis Kit (TAKARA). PCR was performed using Ex Taq polymerase (TAKARA) as directed by the manufacturer. Primer sets used are shown in Supplementary Table I.

\section{Results}

Identification of FAM96B as an interaction partner of E2-2-When we tried to identify HLH-interacting protein(s) by the method of yeast two hybrid system using the HLH domain of Herp2 as a bait, FAM96B was isolated. Composed of 163 amino acids, FAM96B does not possess any known conserved domains._Among HLH proteins examined, FAM96B obviously interacted with E2-2 in COS7 cells (Fig. 1a and data not shown), although the expression of E2-2 was decreased in the presence of FAM96B (see below). This interaction between E2-2 and FAM96B led us to investigate whether E2-2 colocalizes with FAM96B. After MEECs were transfected with Flag-E2-2 and Myc-FAM96B, the subcellular localization was determined by fluorescence microscopy by staining E2-2 with Alexa488-conjugated goat anti-mouse IgG and FAM96B with Alexa555-conjugated goat anti-rabbit IgG. As seen in Fig. 1b, FAM96B could colocalize with E2-2 in the nucleus, which is consistent with an interaction between the two proteins. Id1 has been reported to interact with E2-2 to rescue E2-2-mediated inhibition of angiogenic reaction (8). Since Id1 induced by BMP or VEGF potentiates angiogenesis $(8,21,23,24)$, we tried to test the possibility that the expression of FAM96B is enhanced upon BMP or VEGF stimulation. Instead of BMP stimulation, we infected ALK6ca/HA, which successively transduces BMP signaling into the cells without the ligands, into cells. 
As seen in Fig. 1c, FAM96B mRNA was augmented upon BMP signaling in a similar fashion to Id1. On the other hand, the expression of E2-2 mRNA was not changed upon the ALK6 activation (Suppl. Fig. 1). Furthermore, Id1 mRNA, was induced in endothelial cells by VEGF as expected, whereas VEGF did not enhance the expression of FAM96B transcript (Suppl. Fig. 2). It remains veiled why the stimulation of BMP, but not that of VEGF, accelerated the expression of FAM96B transcript in endothelial cells. We will need further exploration in the future experiments.

Inhibitory effect of FAM96B on E2-2-mediated promoter activity - The MCKpfos-luc reporter construct consisting of four E-box elements has been used to investigate the function of E proteins (18). Our previous studies reported that Id1 and SCL/TAL1, which are interaction partners of E2-2, could counteract E2-2-mediated MCKpfos-luc reporter activity $(7,8)$. To examine the possibility that FAM96B affects the MCKpfos-luc reporter activity induced by E2-2, we transfected a different amount of FAM96B expression vector into the CPAE cells with or without E2-2. FAM96B inhibited the E2-2-mediated activity of this reporter in a dose-dependent manner (Figure 2a) To further confirm that FAM96B influences the transcriptional activity of E2-2, we employed the pGL2b-VEGFR2-luc (-166bp/+267bp) luciferase reporter construct, which E2-2 negatively controls $(7,8)$. Consistent with the result shown in Fig. 2a, FAM96B dose-dependently released the E2-2-mediated inhibition of pGL2b-VEGFR2-luc (-166bp/+267bp) luciferase activity (Fig. 2b). These results demonstrated the possibility that FAM96B interferes with the transcriptional regulation of E2-2.

Activation of EC by FAM96B- E2-2 has been reported to hamper the expression of VEGFR2 in ECs to keep ECs quiescent, whereas Id1 and SCL/TAL1 rescue the 
E2-2-mediated suppression of ECs $(7,8)$. Migration, proliferation, survival, and differentiation in ECs are considerably altered during the process of EC activation. To investigate the effect of FAM96B on EC migration, we infected HUVECs with either GFP- or FAM96B-expressing adenoviruses prior to seeding the ECs on a fibronectin-coated filter (Fig. 3a). We then counted the number of ECs under the chamber. As seen in Figure 3b, FAM96B potentiated the EC migration. Again, the overexpression of FAM96B in HUVECs decreased the E2-2 protein (Fig. 3a). We also tested whether FAM96B affected cell proliferation and network formation in ECs after ECs were infected with either GFP- or FAM96B-expressing adenoviruses. Overexpression of FAM96B in ECs potentiated both the cell growth (Fig. 3c) and the formation of cord-like structures (Fig. 3d and 3e). Thus, these observations indicate that in contrast to E2-2, FAM96B has the ability to keep ECs active.

Effect of FAM96B on the expression of E2-2- Because ectopic FAM96B seems to influence the expression of E2-2 protein in COS7 cells (Fig. 1a) and HUVECs (Fig. 3a), we further investigated whether the expression of E2-2 protein is attenuated by the ectopic expression of FAM96B. When Myc-FAM96B was overexpressed with Flag-E2-2 in COS7 cells, the expression of Flag-tagged E2-2 was diminished with the increasing expression of Myc-FAM96B (Fig. 4a), and vice versa (Fig. 4b). Thus, the decrease of E2-2 expression mediated by FAM96B was not dependent on the tags. Furthermore, the ectopic expression of FAM96B decreased the endogenous E2-2 protein in NIH3T3 cells (Fig. 4c). Since E12/47 belongs to the E protein family, we also tested if FAM96B affected the expression of the E47 protein. Expectedly, the expression of E47 protein was also decreased by FAM96B to the same extent as that of E2-2. On the other hand, the 
expression of neither Smad2 (Fig. 4d) nor c-myc (Suppl. Fig. 3) was altered by FAM96B. Therefore, this finding strongly suggests that FAM96B is able to specifically affect the protein expression of the E protein family.

Exploration of the functional domain in FAM96B-To find the functional domain in FAM96B required for the decrease of E2-2 protein expression, we made two FAM96B mutants, termed FAM96B(42-121) and FAM96B $\Delta$ (42-121) (Fig. 5a). Then, we explored which part(s) of FAM96A can affect the expression of E2-2 protein. As seen in Fig. 5b, FAM96B(42-121), but not FAM96B $\Delta$ (42-121), could decrease the expression of E2-2 even if the expression of FAM96B (42-121) was relatively lower than that of FAM96B $\Delta$ (42-121). Collectively, the domain from $\mathrm{Asp}^{42}$ to $\mathrm{Ile}^{121}$ in FAM96B is necessary for FAM96B to decrease the expression of E2-2.

\section{Discussion}

E protein family members such as E2-2 and E12/47 are widely expressed and act as either transcriptional activators or transcriptional repressors dependent on the partner(s) that the E protein family can interact with (25-28). E proteins are known to play key roles in the regulation of cell proliferation and differentiation. For example, E2-2 and E12/47 play critical roles in B-cell development and perturbed T-cell development (29-31). In our previous studies, E2-2 kept ECs quiescent, whereas the HLH proteins Id1 and SCL/TAL1 were capable of overcoming the inhibitory action of E2-2 on EC functions $(7,8)$. In the present study, FAM96B, which is not a member of the HLH protein family, was found to decrease the expression of E2-2 protein in cells so as to perturb E2-2-mediated cellular responses such as transcriptional activities and EC 
quiescence. The experiments using FAM96B mutants predicted that the middle region of FAM96B is critical for it to exhibit limited expression of E2-2 protein. Although FAM96B does not have any known conserved domains, the middle region of FAM96B shows similarity to 'domain of unknown function 59 (DUF59)' in prokaryotes. Thus, to the best of our knowledge, this is the first report to define the functional significance of DUF59.

Since a number of proteins in cells can be degraded through either the proteasomal or the lysosomal pathways (32), we tried to investigate whether FAM96B can also decrease the expression of the E2-2 protein via these pathways. However, the decrease of E2-2 expression mediated by FAM96B was not rescued by inhibitors specific for either proteasomes or lysosomes (Suppl. Fig. 4 and 5). As another possibility, we checked the transcript of E2-2 with or without FAM96B in either the absence or the presence of actinomycin D. However, the transcript of E2-2 was not altered by FAM96B (data not shown). These results indicate the possibility that FAM96B can activate a certain protease(s) that targets E2-2. Because we currently do not know the exact mechanism by which FAM96B can diminish the expression of the E2-2 protein, several possibilities still need to be explored. Furthermore, it is interesting to analyze that FAM96B can be regulated at a transcriptional and/or a translational level by itself.

In a previous study, we found that E2-2 interfered with EC activation while its interaction partners Id1 and SCL/TAL1 overcame the function of E2-2 and activated ECs $(7,8)$. Besides, FAM96B was found as one of BMP-inducing genes like Id1 in our present study. Therefore, we assumed that FAM96B could also potentiate the function of ECs. Indeed, we were convinced that FAM96B can promote EC activation. Importantly, the 
expression of endogenous E2-2 protein was diminished by FAM96B, demonstrating that FAM96B restricts the expression of E2-2 protein to control E2-2-mediated cellular responses in ECs. Our hypothesis can be further supported by the evidence that FAM96B could rescue the E2-2-mediated suppression of the VEGFR2 promoter. Thus, it is high possibility that the protein expression of VEGFR2 is influenced by FAM96B. This possibility will be tested in our further investigation. Since E2-2 is ubiquitously expressed in cells, it will be interesting to know if FAM96B regulates the function of E2-2 via the control of E2-2 expression in cell types other than ECs. Our preliminary study indicated that FAM96B could not compete with Id1 for the interaction with E2-2 despite the fact that both Id1 and FAM96B can associate with E2-2. Therefore, the function of E2-2 might be fine-tuned at different steps within cells via the regulation of its protein expression, its DNA binding ability, and so forth.

In conclusion, we discovered a novel mechanism by which FAM96B impairs the expression of E2-2 protein in ECs to potentiate EC activation. The identification of compounds that bolster E2-2 activity via the activation of FAM96B in ECs may provide an exciting opportunity to modulate tumor angiogenesis for therapeutic intervention. 


\section{Acknowledgements}

This research was supported by Grants-in-aid for Scientific Research (17390073, 20012007 and 21590328) (M.K. and S.I.) from the Ministry of Education, Culture, Sports, Science, and Technology of Japan; a Grant-in-Aid for JSPS Fellows from the Japan Society for the Promotion of Science (W. Y. and F. I.); the Takeda Science Foundation (S.I.); and the Naito Foundation (S.I.). We thank Ms. F. Miyamasu for excellent English proofreading.

\section{Disclosure Statement}

The authors have no conflict of interest. 


\section{References}

1. Henthorn P, Kiledjian M, Kadesch T. Two distinct transcription factors that bind the immunoglobulin enhancer $\mu$-E5/א-E2 motif. Science, 1990; 247: 467-70.

2. Lazorchak A, Jones ME, Zhuang Y. New insights into E-protein function in lymphocyte development. Trends Immunol, 2005; 26: 334-38.

3. Sun XH. Multitasking of helix-loop-helix proteins in lymphopoiesis. Adv Immunol, 2004; 84: 43-77.

4. Murre C. Helix-loop-helix proteins and lymphocyte development. Nat Immunol, 2005; 6: 1079-86.

5. Einarson MB, Chao MV. Regulation of Id1 and its association with basic helix-loop-helix proteins during nerve growth factor-induced differentiation of PC12 cells. Mol Cell Biol, 1995; 15: 4175-83.

6. Puri PL, Sartorelli V. Regulation of muscle regulatory factors by DNA-binding, interacting proteins, and post-transcriptional modifications. J Cell Physiol, 2000; 185: 155-73.

7. Tanaka A, Itoh F, Itoh S, Kato M. TAL1/SCL relieves the E2-2-mediated repression of VEGFR2 promoter activity. J Biochem, 2009; 145: 129-35.

8. Tanaka A., Itoh F., Nishiyama K et al. Inhibition of endothelial cell activation by bHLH protein E2-2 and its impairment of angiogenesis. Blood, 2010; 115: 4138-47.

9. Deleuze V, Chalhoub E, El-Hajj R, et al. TAL-1/SCL and its partners E47 and LMO2 up-regulate VE-cadherin expression in endothelial cells. Mol Cell Biol, 2007; 27: 2687-97.

10. Benezra R, Davis RL, Lassar A et al. Id: a negative regulator of helix-loop-helix 
DNA binding proteins. Control of terminal myogenic differentiation. Cell, 1990; 61: 49-59.

11. Qiu Y, Sharma A, Stein R. p300 mediates transcriptional stimulation by the basic helix-loop-helix activators of the insulin gene. Mol Cell Biol, 1998: 18: 2957-64.

12. Bradney C, Hjelmeland M, Komatsu Y, Yoshida M, Yao TP, Zhuang Y. Regulation of E2A activities by histone acetyltransferases in B lymphocyte development. J Biol Chem, 2003; 278: 2370-6.

13. Zhang J, Kalkum M, Yamamura S, Chait BT, Roeder RG. E protein silencing by the leukemogenic AML1-ETO fusion protein. Science, 2004; 305:1286-9.

14. Sloan SR, Shen CP, McCarrick-Walmsley R, Kadesch T. Phosphorylation of E47 as a potential determinant of B-cell-specific activity. Mol Cell Biol, 1996; 16: 6900-8.

15. Carmeliet P. Angiogenesis in life, disease and medicine. Nature, 2005; 438: 932-6.

16. Adams, RH, Alitalo, K. Molecular regulation of angiogenesis and lymphangiogenesis. Nat Mol Cell Bio Rev, 2007; 8: 464-78.

17. Goldman LA, Cutrone EC, Kotenko SV, Krause CD, Langer JA. Modifications of vectors pEF-BOS, pcDNA1 and pcDNA3 result in improved convenience and expression. Biotechniques, 1996; 21: 1013-5.

18. Sigvardsson M. Overlapping expression of early B-cell factor and basic helix-loop-helix proteins as a mechanism to dictate B-lineage-specific activity of the $\lambda 5$ promoter. Mol Cell Biol, 2000; 20: 3640-54.

19. Holderfield MT, Henderson Anderson AM, Kokubo H, et al. HESR1/CHF2 suppresses VEGFR2 transcription independent of binding to E-boxes. Biochem Biophys Res Commun, 2006; 346: 637-48. 
20. He T-C, Zhou S, da Costa LT, Yu J, Kinzler KW, Vogelstein B. A simplified system for generating recombinant adenoviruses. Proc Natl Acad Sci USA, 1998; 95: 2509-14.

21. Goumans M-J, Valdimarsdottir G, Itoh S, Rosendahl A, Sideras P, ten Dijke P. Balancing the activation state of the endothelium via two distinct TGF $\beta$ type I receptors. EMBO J, 2002; 21: 1743-53.

22. Takezawa T, Takenouchi T, Imai K, Takahashi T, Hashizume K. Cell culture on thin tissue sections commonly prepared for histopathology. FASEB J, 2002; 16: 1847-9.

23. Korchynskyi O, ten Dijke P. Identification and functional characterization of distinct critically important bone morphogenetic protein-specific response elements in the Id1 promoter. J Biol Chem, 2002; 277: 4883-91.

24. Sakurai D, Tsuchiya N, Yamaguchi A, Okaji Y, Tsuno NH, Kobata T, Takahashi K, Tokunaga K. Crucial role of inhibitor of DNA binding/differentiation in the vascular endothelial growth factor-induced activation and angiogenic processes of human endothelial cells. J Immunol, 2004; 173: 5801-9.

25. Lemercier C, To RQ, Carrasco RA, Konieczny SF. The basic helix-loop-helix transcription factor Mist1 functions as a transcriptional repressor of myoD. EMBO J, 1998; 17: 1412-22.

26. Murayama A, Kim MS, Yanagisawa J, Takeyama K, Kato S. Transrepression by a liganded nuclear receptor via a bHLH activator through co-regulator switching. EMBO J, 2004; 23: 1598-608.

27. Zhang J, Kalkum M, Yamamura S, Chait BT, Roeder RG. E protein silencing by the leukemogenic AML1-ETO fusion protein. Science, 2004; 305: 1286-9. 
28. Goardon N, Lambert JA, Rodriguez P et al. ETO2 coordinates cellular proliferation and differentiation during erythropoiesis. EMBO J, 2006; 25: 357-66.

29. Zhuang Y, Soriano P, Weintraub H. The helix-loop-helix gene E2A is required for B cell formation. Cell, 1994; 79: 875-84.

30. Bain G, Maandag EC, Izon DJ, et al. E2A proteins are required for proper B cell development and initiation of immunoglobulin gene rearrangements. Cell, 1994; 79: 885-92.

31. Bain G, Quong MW, Soloff RS, Hedrick SM, Murre C. Thymocyte maturation is regulated by the activity of the helix-loop-helix protein, E47. J Exp Med, 1999; 190: 1605-16.

32. Clague MJ, Urbé S. Ubiquitin: same molecule, different degradation pathways. Cell, 2010; 143: 682-5. 


\section{Figure legends}

Fig. 1 Association between FAM96B and E2-2. (a) Interaction of Myc-E2-2 with Flag-FAM96B. Myc-E2-2 was cotransfected with Flag-FAM96B. Immnunoprecipitations were carried out with anti-Flag M5 antibody, and coimmunoprecipitated E2-2 was detected by Western blotting with anti-Myc9E10 antibody (upper panel). The expressions of Myc-E2-2 and Falg-FAM96B were evaluated by using anti-Myc9E10 antibody (middle panel) and anti-Flag M5 antibody (lower panel), respectively. (b) FAM96B colocalizes with E2-2 in the nucleus. MEECs were transiently transfected with Flag-FAM96B and Myc-E2-2. Flag-FAM96B and Myc-E2-2 were visualized with red and green, respectively, as described in the text. Colocalization of E2-2 with FAM96B appears as yellow. Nuclei were stained with DAPI. (c) Induction of FAM96B mRNA upon ALK6 activation. MEECs were infected with either LacZ- or ALK6ca-expressing adenoviruses. Then, RT-PCR was performed using sets of two specific primers for FAM96B (top panel), Id1 ( $2^{\text {nd }}$ panel), Smad6 ( $3^{\text {rd }}$ panel) and $\beta$-actin $\left(4^{\text {th }}\right.$ panel). Both the expressions of Id1 and Smad6 were used as known BMP-inducible genes, whereas $\beta$-actin was used as a loading control. The expression of ALK6ca was detected with anti-HA antibody (bottom panel).

Fig. 2 FAM96B counteracts E2-2-mediated luciferase activity. (a) FAM96B inhibits E2-2-induced MCKpfos-luc activity. CPAEs were transfected with MCKpfos-luc and different amounts of FAM96B and E2-2. (b) FAM96B relieves the inhibition of 
pGL2b-VEGFR2-luc (-166bp/+267bp) activity by E2-2. CPAEs were transfected with pGL2b-VEGFR2-luc (-166bp/+267bp), FAM96B, and E2-2.

Fig. 3 FAM96B potentiates EC activation. (a) The decrease of E2-2 expression in ECs. After the adenoviral infection, total lysates were prepared. Then, Western blotting was performed. Expression of endogenous E2-2 was evaluated with anti-E2-2 antibody (top panel). Myc-FAM96B was detected with anti-Myc9E10 antibody ( $2^{\text {nd }}$ panel). As internal controls, GFP ( $3^{\text {rd }}$ panel) and $\beta$-actin (lower panel) were visualized by using anti-GFP antibody and anti- $\beta$-actin antibody, respectively. (b) FAM96B activates EC migration. After adenoviral infection, HUVECs were seeded on the upper membrane of the Boyden chamber. After $24 \mathrm{~h}$, cells were stained with crystal violet and the number of transmigrated cells was counted. (c) FAM96B enhances EC proliferation. After adenoviral infection, cells were labeled with $\left[{ }^{3} \mathrm{H}\right]$-thymidine. Then, the ${ }^{3} \mathrm{H}$ radioactivity incorporated into DNA was determined by liquid scintillation counting. (d) FAM96B potentiates the formation of cord-like structures on the Matrigel. Forty hours after adenoviral infection, HUVECs were seeded on the Matrigel. The images at 6 and $12 \mathrm{~h}$ time points are shown. The $t$ test was adapted to analyze significant difference between GFP- and FAM96B-infected cells. (e) Areas of cord-like structures in Fig. 3d were measured by Image $\mathrm{J}$.

Fig. 4 Effect of FAM96B on the expression of E2-2. (a, b) Decrease of E2-2 protein by FAM96B. Different amounts of Myc-FAM96B (a) and Flag-FAM96B (b) were cotransfected with Flag-E2-2 (a) and Myc-E2-2 (b), respectively. After preparation of 
total lysates, the expressions of Flag-E2-2 (a) and Myc-E2-2 (b) were evaluated by using anti-Flag M5 antibody and anti-Myc9E10 antibody, respectively (upper panel). The expression of FAM96B was detected with anti-Myc9E10 (a) and anti-Flag M5 antibodies (b) (middle panel). As a loading control, the expression of $\beta$-actin was observed with anti- $\beta$-actin antibody (lower panel). (c) Decrease of endogenous E2-2 protein by FAM96B. NIH3T3 cells were transfected with a different amount of Myc-FAM96B. After preparation of total lysates, endogenous E2-2 was detected with anti-E2-2 antibody (upper lane). The expression of FAM96B was detected with anti-Myc9E10 (middle panel). As a loading control, the expression of $\beta$-actin was observed with anti- $\beta$-actin antibody (lower panel). (d) Decrease of E47 protein by FAM96B. The different amounts of Myc-FAM96B were cotransfected with Flag-E2-2, Flag-E47, or Flag-Smad2. After preparation of total lysates, the expressions of Flag-E2-2 (top), Flag-E47 (middle), and Flag-Smad2 (lower) were evaluated by using anti-Flag M5 antibody (left panels). The expression of FAM96B was detected with anti-Myc9E10 antibody (right panel).

\section{Fig. 5 The middle domain in FAM96B is critical for its activity to decrease E2-2} protein. (a) Mutants of FAM96B. (b) Degradation of E2-2 by FAM96B (42-121). The different amounts of either Myc-FAM96B(42-121) or Myc-FAM96BA(42-121) were transfected with Flag-E2-2. After preparation of total lysates, Flag-E2-2 was detected with anti-Flag M5 antibody (upper lane). The expression of Myc-FAM96B(42-121) or Myc-FAM96BA(42-121) was detected with anti-Myc9E10 (middle panel). Because the expression of Myc-FAM96B (42-121) was lower than that of Myc-FAM96B 4 (42-121), 
two photos were taken, one with short exposure and one with long exposure. As a loading control, the expression of $\beta$-actin was observed with anti- $\beta$-actin antibody (lower panel). 


\section{A list of Supporting Information}

Additional Supporting Information may be found in the online version of this article.

Supplementary Table: PCR primers to amplify mouse cDNAs.

Supplementary Figure legends: 2 pages

Suppl. Fig. 1: Expression of E2-2 mRNA upon ALK6 activation.

Suppl. Fig. 2: Effect of FAM96B on expression of c-myc protein.

Suppl. Fig. 3: Effect of proteasome inhibitors on FAM96B-mediated decrease of E2-2

protein.

Suppl. Fig. 4: Effect of a lysosome inhibitor on FAM96B-mediated decrease of E2-2 protein. 
Fig. 1 (Yang et al.)

(b)

(a)

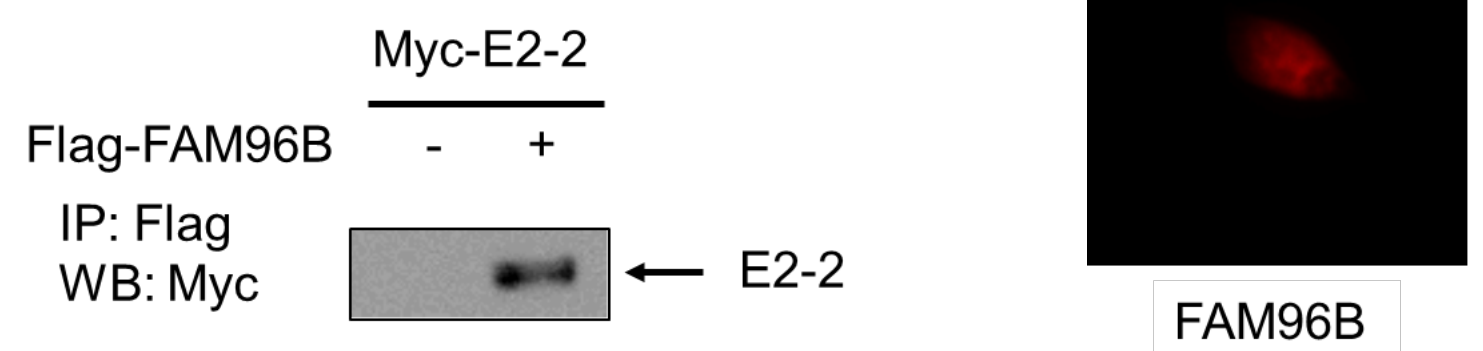

E2-2

WB: Myc

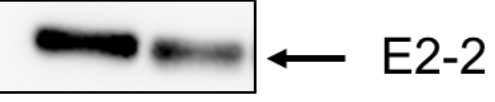

WB: Flag
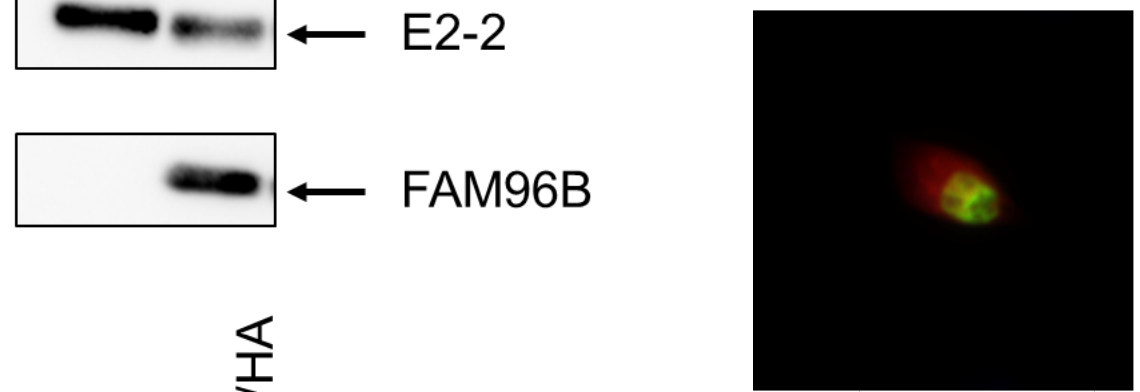

Merge

DAPI

(c)

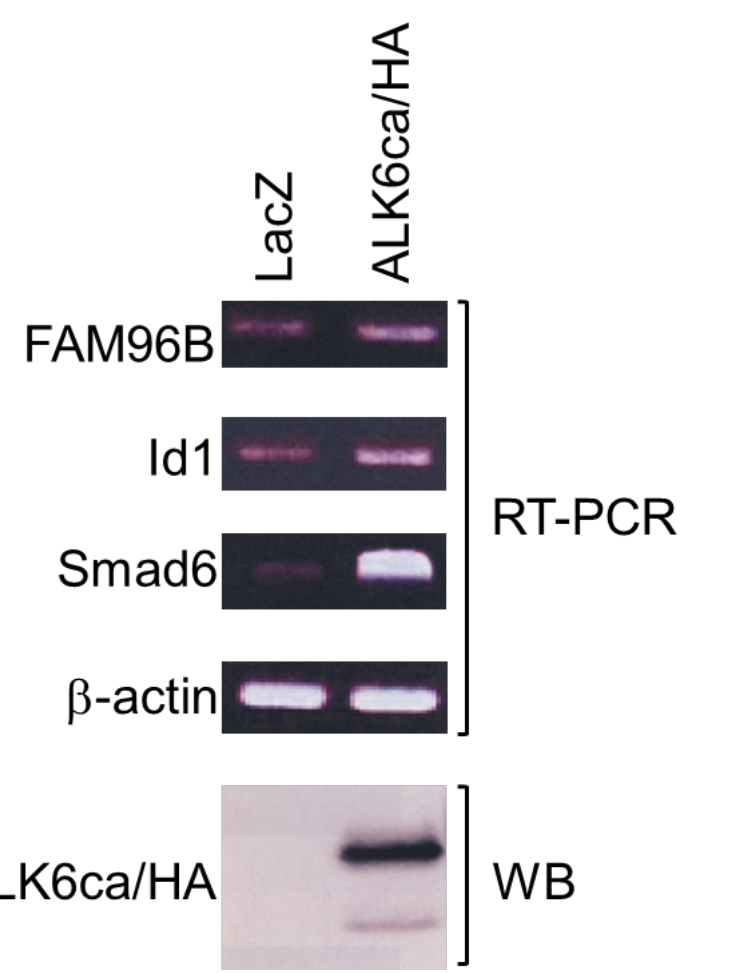


Fig. 2 (Yang et al.)
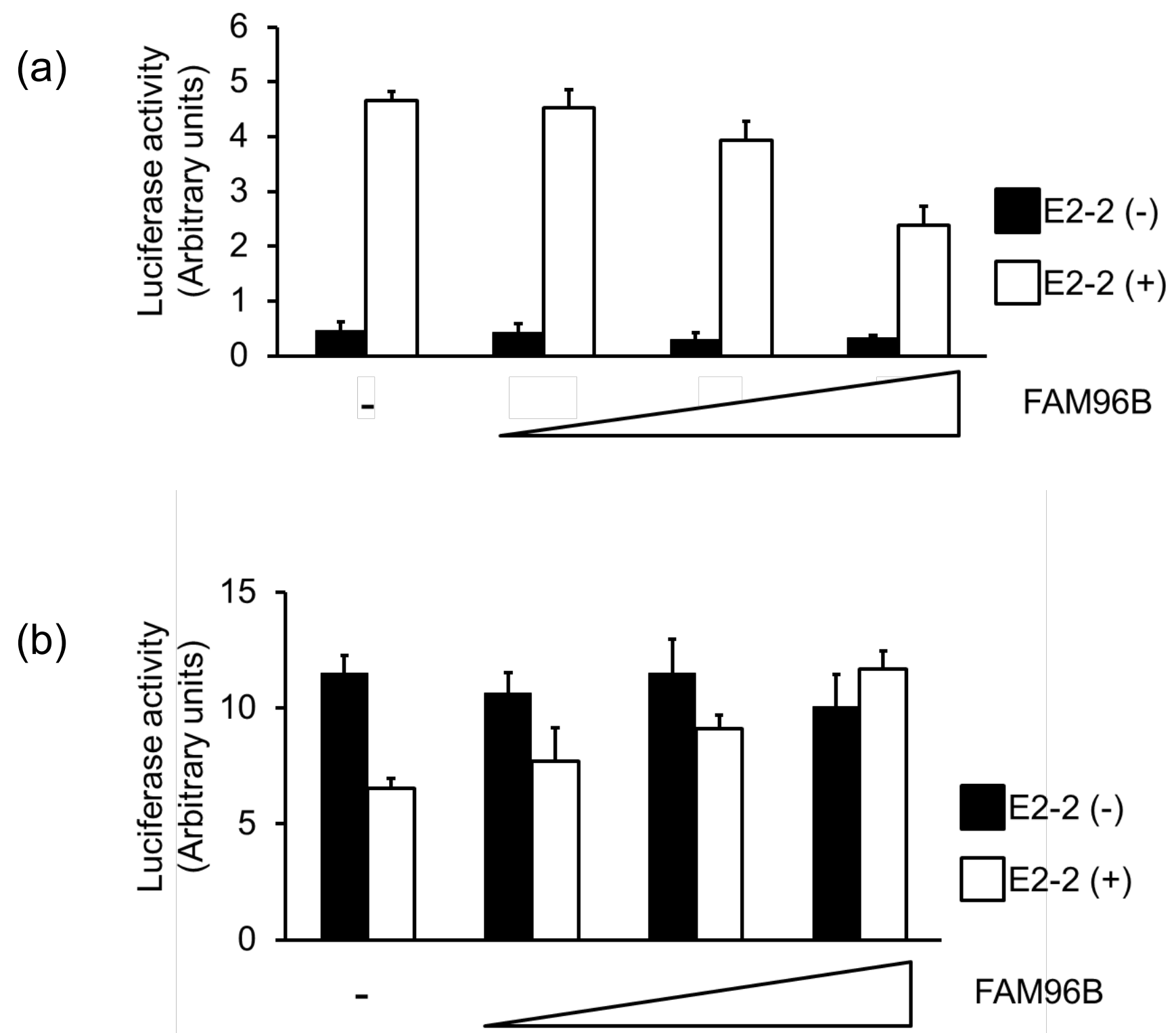
Fig. 4 (Yang et al.)
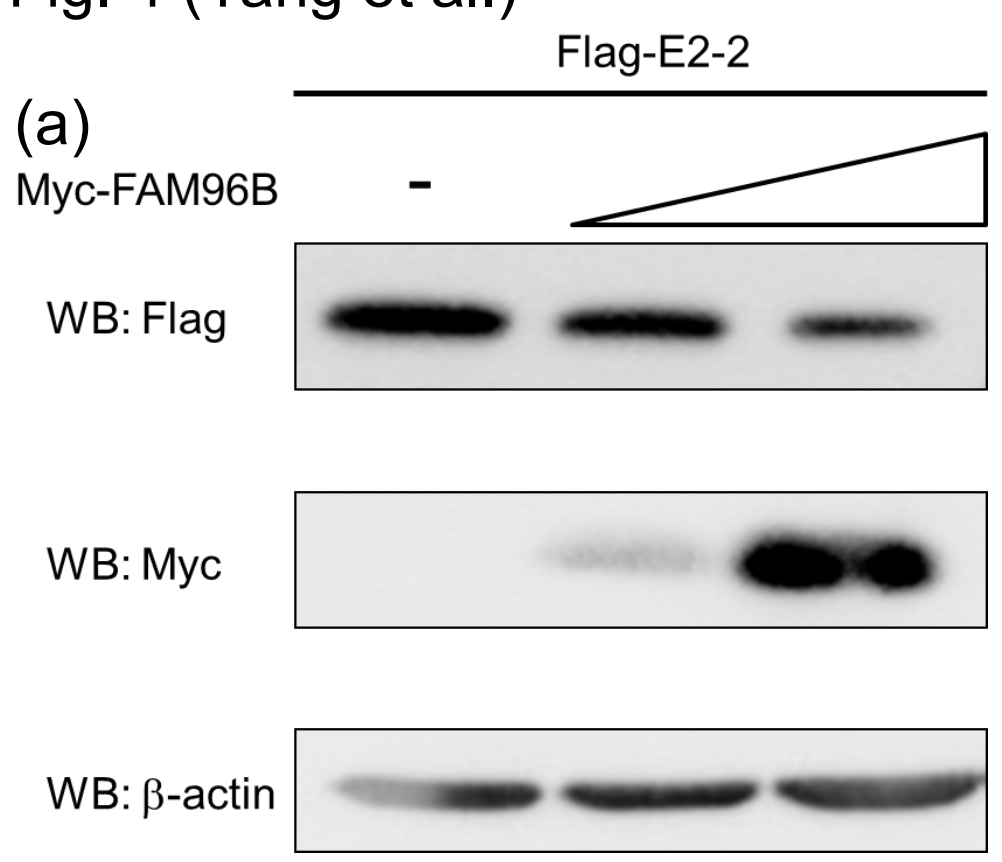

(c)
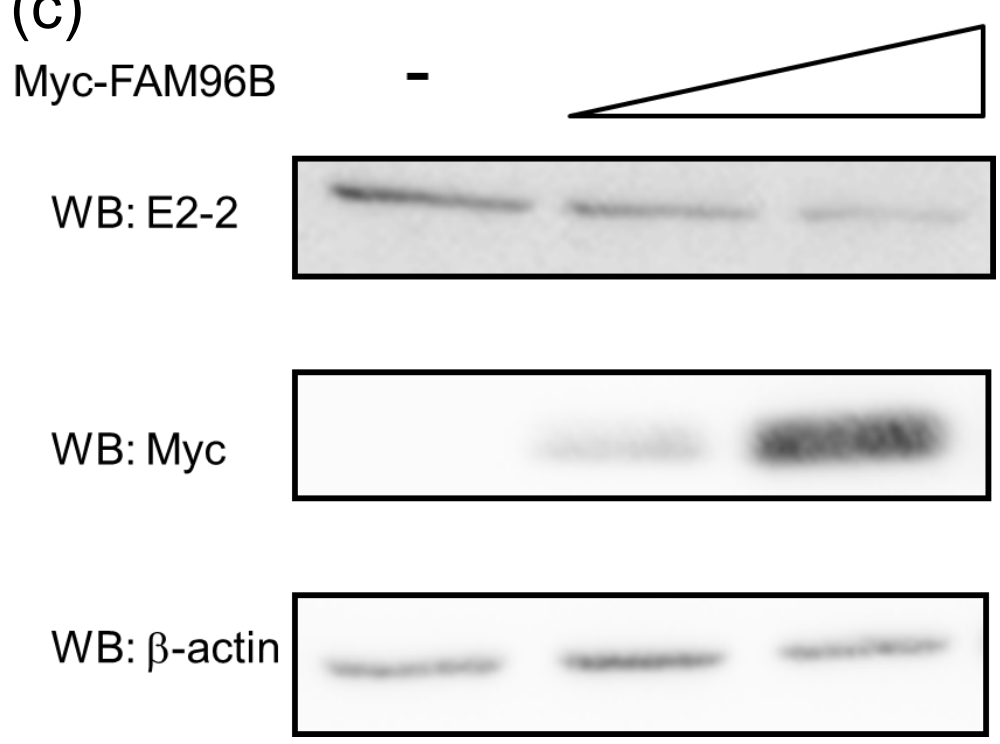

(b)

Myc-E2-2
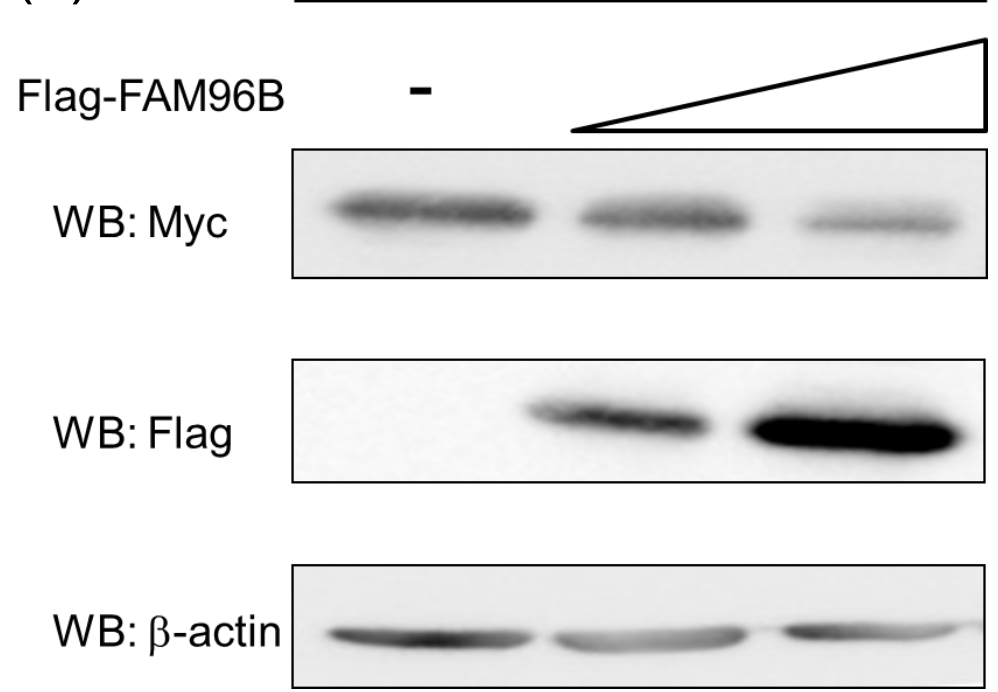

(d)

WB: Flag

WB: Myc
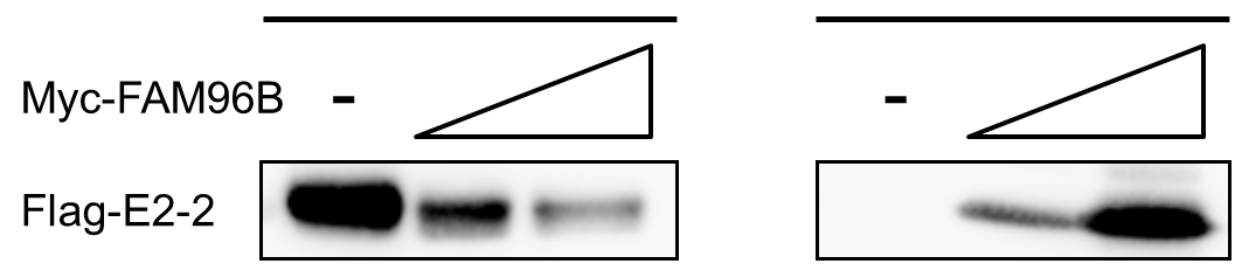

Flag-E47
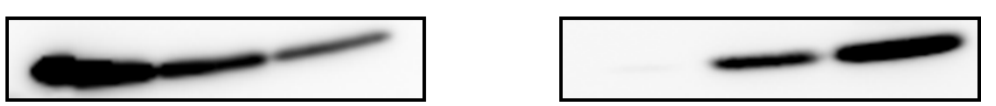

Flag-Smad2 
Fig. 5 (Yang et al.)

(a)

FAM96B

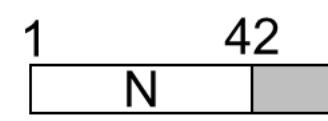

\begin{tabular}{c|c}
$121 \quad 163$ \\
\hline
\end{tabular}

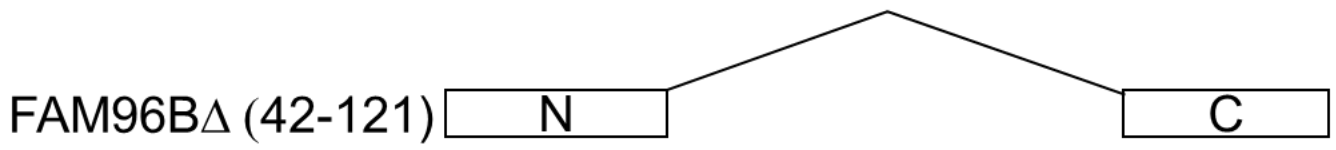

FAM96B(42-121)

Flag-E2-2

(b)
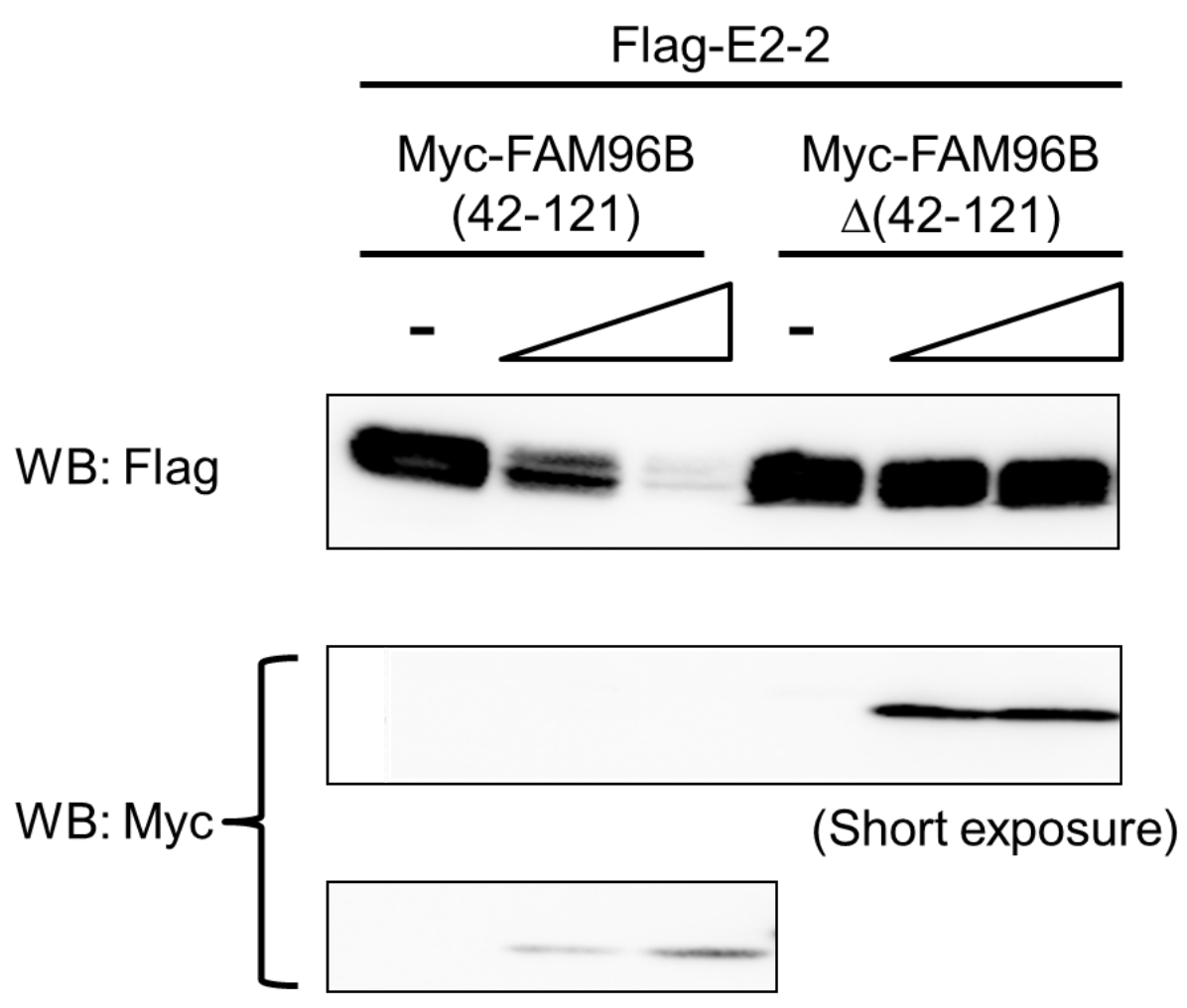

(Long exposure)

WB: $\beta$-actin 


\section{Supplementary Figure legends}

Suppl. Fig. 1. Expression of E2-2 mRNA upon ALK6 activation. Mouse embryonic endothelial cells (MEECs) were infected with either LacZ- or ALK6ca-expressing adenoviruses. Then, RT-PCR was performed using sets of two specific primers for E2-2 (5’-GTGGCCATTGAAGGTTTGTG-3’/3'-TCGTGAAAGGGATCGAGGAA-5’) (top panel), Smad7 (5’-TCCAGATGCTGTACCTTCCT-3’/3’-AAATGTTGGCGTCGTCAATG-5') (middle panel) and $\beta$-actin (5’-TGAACCCTAAGGCCAACCGTG-3'/3’-GCTCATAGCTCTTCTCCAGGG-5') (bottom panel). Smad7 was used as a known BMP-inducible gene, whereas $\beta$-actin was used as a loading control.

Suppl. Fig. 2. Effect of VEGF on expression of FAM96B. MEECs were stimulated with VEGF for 0, 1, 2 and $6 \mathrm{~h}$. Then, RT-PCR was performed using sets of two specific primers for VEGFR2 (5’-AGCTGTCGCTCTGTGGTTCT-3’/3’-CGTTTTGTGAGTGGTAAGGG-5’) (top panel). Other primer sets were described in Suppl. Table I and Suppl. Fig. 1. 
Suppl. Fig. 3. Effect of FAM96B on expression of c-myc protein. The different amounts of Myc-FAM96B were cotransfected with Flag-c-myc in COS7 cells. After preparation of total lysates, the expression of Flag-c-myc was evaluated by using anti-Flag M5 antibody (upper panel). The expression of FAM96B was detected by using anti-Myc9E10 antibody (middle panel). As a loading control, the expression of $\beta$-actin was observed with anti- $\beta$-actin antibody (lower panel).

\section{Suppl. Fig, 4. Effect of proteasome inhibitors on FAM96B-mediated decrease of}

E2-2 protein. Different amounts of Myc-FAM96B were cotransfected with Flag-E2-2 in COS7 cells. Four hours before the preparation of total lysates, proteasome inhibitors (10 $\mu \mathrm{M}$ MG-132 and $2 \mu \mathrm{M}$ lactacystin) were added to the medium. DMSO was used as a mock stimulation. The expression of Flag-E2-2 was evaluated by using anti-Flag M5 antibody (upper panel). The expression of FAM96B was detected by using anti-Myc9E10 antibody (middle panel). As a loading control, the expression of $\beta$-actin was observed with anti- $\beta$-actin antibody (lower panel).

\section{Suppl. Fig. 5. Effect of a lysosome inhibitor on FAM96B-mediated decrease of E2-2}

protein. Different amounts of Myc-FAM96B were cotransfected with Flag-E2-2 in 
COS7 cells. Four hours before the preparation of total lysates, a lysosome inhibitor (50

$\mu \mathrm{g} / \mathrm{mL}$ chloroquine) was added to the medium. The expression of Flag-E2-2 was

evaluated by using anti-Flag M5 (upper panel) antibody. The expression of FAM96B was

detected by using anti-Myc9E10 antibody (middle panel). As a loading control, the

expression of $\beta$-actin was observed with anti- $\beta$-actin antibody (lower panel). 
Suppl. Fig. 1

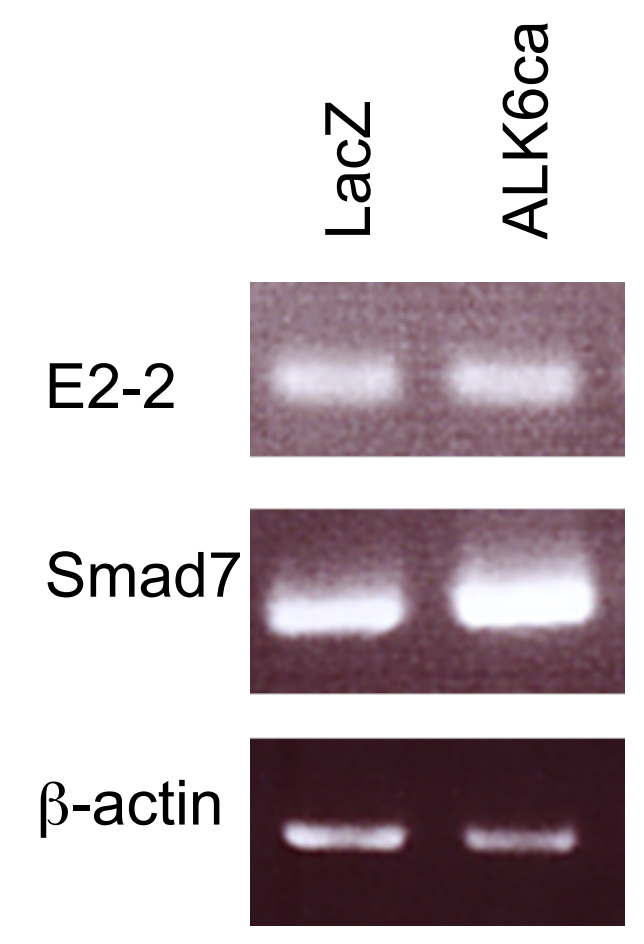


Suppl. Fig. 2
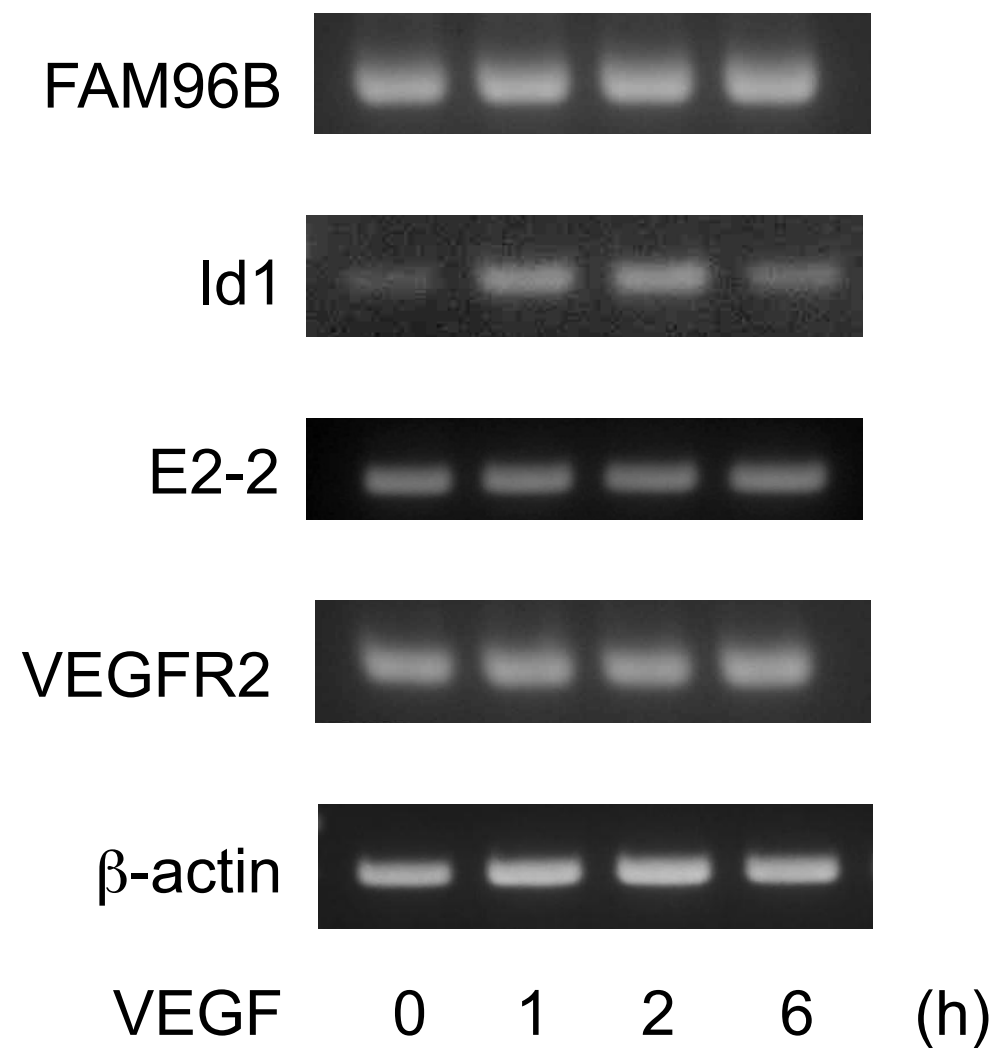
Suppl. Fig. 3

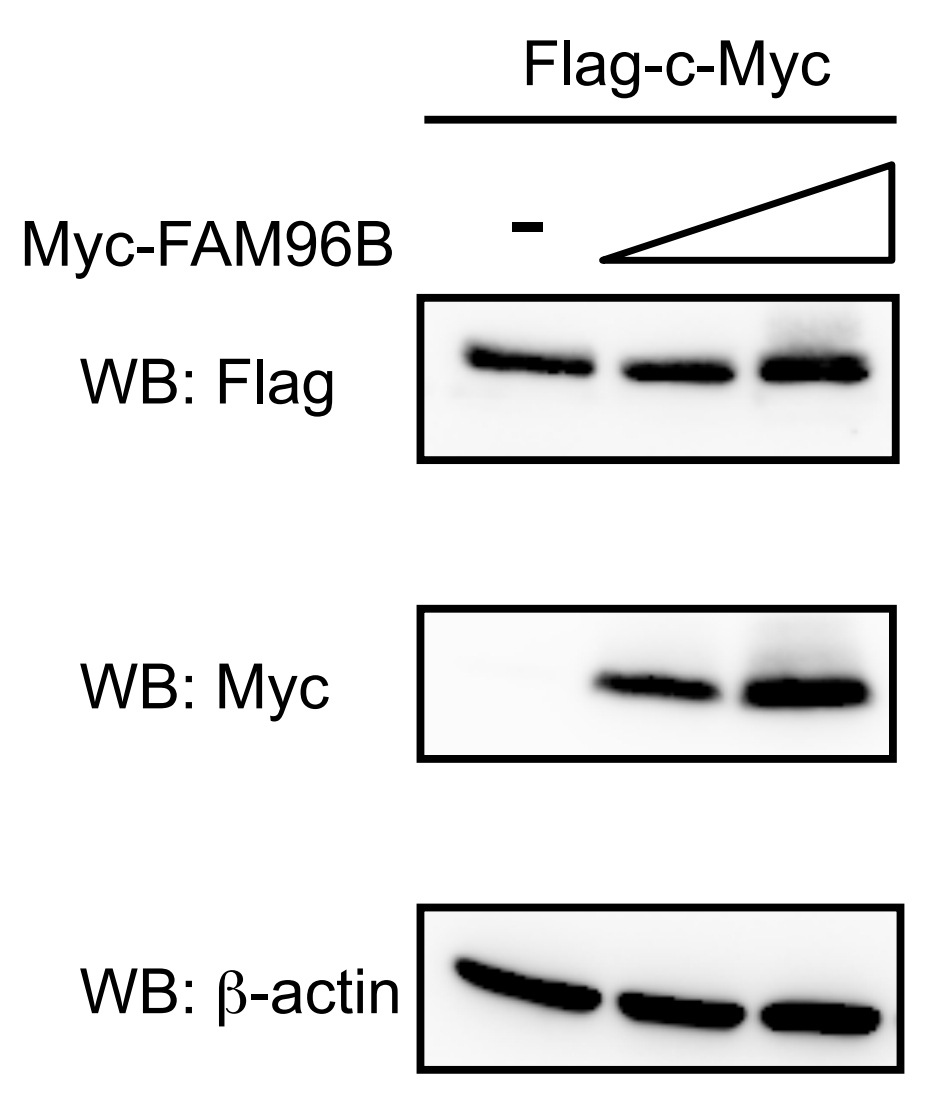


Suppl. Fig. 4
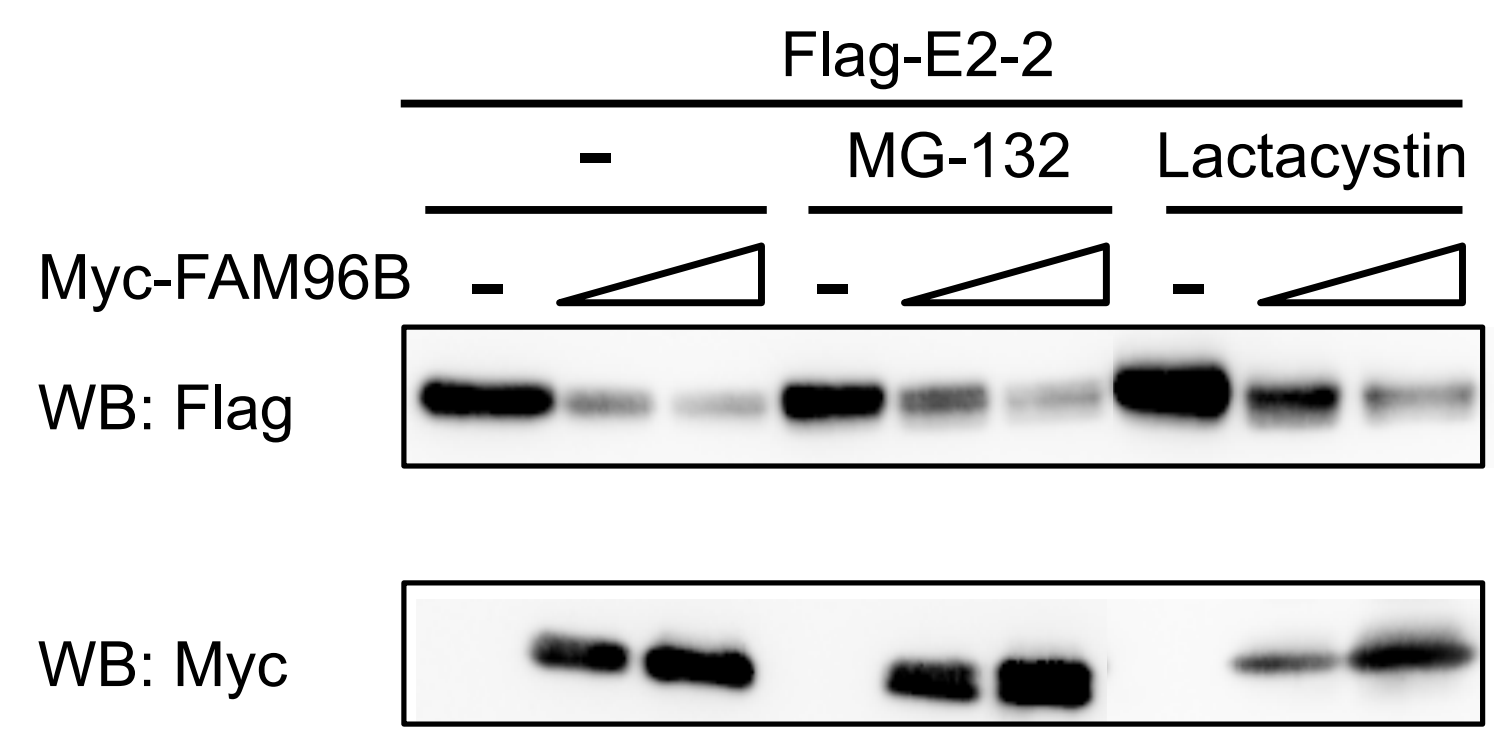

WB: $\beta$-actin 
Suppl. Fig. 5

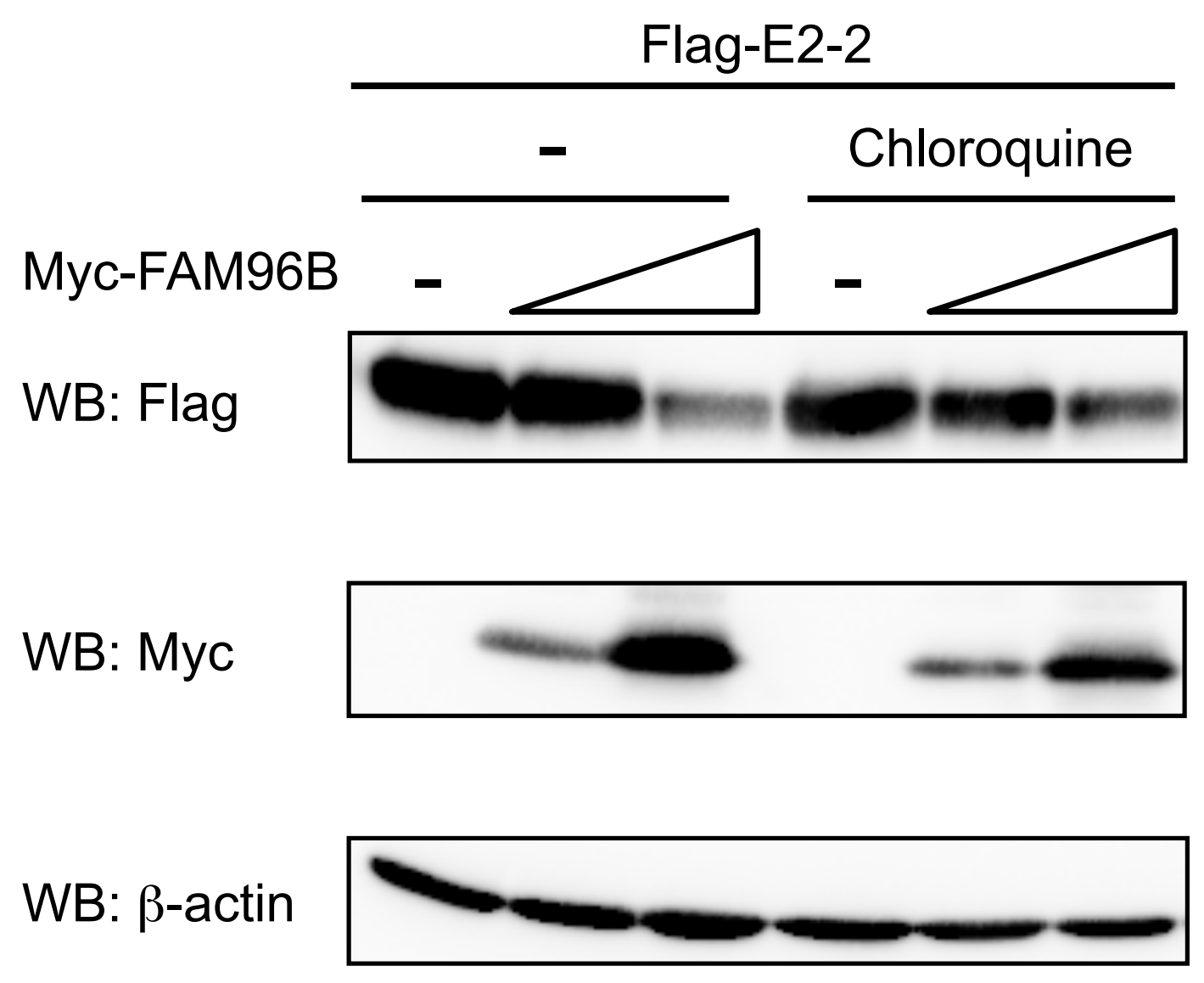




\section{Suppl. Table I PCR primers to amplify mouse cDNAs}

\begin{tabular}{|l|l|l|}
\hline \multicolumn{1}{|c|}{ Human CDNA } & & Sequence \\
\hline FAM96B & $(+)$ & CGAGGAAGATGAGGAAGTGC \\
& $(-)$ & CGCTCCTTATCTGCAAGTTG \\
\hline Id1 & $(+)$ & ACTTGGTCTGTCGGAGCAAA \\
& $(-)$ & CAGCGACACAACTAGCGATC \\
\hline Smad6 & $(+)$ & TGTTGCAACCCCTACCACTT \\
& $(-)$ & GTCGTTCTAGCCAAAACCGT \\
\hline$\beta$-actin & $(+)$ & GCTCATAGCTCTTCTCCAGGG \\
& $(-)$ & GTGCCAACCGGAATCCCAAGT \\
\hline
\end{tabular}

$L$ : length of PCR fragment; AT: annealing temperature 